\title{
Histórias em jogo: notas sobre videogames e percursos narrativos em day of the tentacle
}

Jorge Adeodato $\mathrm{Jr}^{1}$

Ricardo Jorge de Lucena Lucas ${ }^{2}$

\section{Resumo}

Jogos eletrônicos constituem elementos de uma cultura popular recente e possuem, entre o público jovem, enorme potência comunicativa. No entanto, pouco foi produzido em termos de análise acadêmica sobre as possibilidades narrativas deste universo; fato curioso, uma vez que videogames permitem análises profícuas dentro de áreas que estimulem diálogos entre linguagens artísticas e contextos culturais diversos. Este trabalho visa ressaltar, dentro do jogo Day of the Tentacle (1993; 2016), características gerais do gênero adventure e as formas como nele se desenvolvem processos de referenciação a outros itens da cultura pop (cinema, desenhos animados, e personagens arquetípicas da literatura de terror e de ficção científica). Recorreremos, para fins de análise, à proposições de críticos da cultura recente (JAMESON, 1991) e teóricos do Game Studies (GALLOWAY, 2006; NEWMAN, 2004 e 2008).

Palavras-chave: Narrativas contemporâneas intermidiais. Literatura intermídia. Contemporaneidade.

\section{STORIES AT STAKE: NOTES ON VIDEOGAMES AND NARRATIVE DEVICES IN DAY OF THE TENTACLE}

\begin{abstract}
Electronic games are a strong element in contemporary culture due to its powerful communicative potential among youngsters. However, little has been produced in terms of critical and academic analysis in Brazil concerning the narrative processes of this particular universe; a surprising fact, since videogames may stand as an interesting topic of analysis within areas which stimulate dialogues between cultural and artistic contexts. This work aims to highlight, in Day of the Tentacle $(1993,2016)$, narrative dynamics in the adventure game genre and the ways by which it applies references to other cultural artifacts (cinema, cartoons and archetypal characters of the literature of terror and Science fiction). For the sake of analysis, propositions by recent cultural critics (JAMESON, 1991) and Game Studies theorists (GALLOWAY, 2006; NEWMAN, 2004 and 2008) will be used.
\end{abstract}

Keywords: Videogames. Contemporary narratives. Intermedia and literature. Contemporaneity. Introdução

1 Programa de Pós-graduação, Universidade Federal do Ceará. E-mail: jorgeadeodato@yahoo.com.br

2 Doutorado em Comunicação Social. Professor do Programa de Pós-graduação da Universidade Federal do Ceará. E-mail: ricardo.jorge@yahoo.com> 
Videogames têm sido, há algumas décadas, elementos de presença relevante dentro do contexto da cultura popular. De acordo com Galloway (2006, p. xi), jogos eletrônicos "têm ocupado uma posição central na cultura de massa por mais de vinte anos, e surpreendentemente poucas obras ocupam-se de uma análise crítica desse meio"3. É possível argumentar que possuam influência semelhante à televisão e à internet em meio ao público jovem; talvez até algo mais direta, uma vez que games têm a capacidade de não apenas entreter, mas também de formar comunidades segmentadas dispostas a interpretar, gerar e repassar informações acerca deste artefato cultural (NEWMAN, 2008, p. 5). O vasto investimento financeiro por parte da indústria pode ser justificado, óbvio, por um vantajoso retorno. Mas o que nos videogames os tornam tão populares, e o que no debate em torno deles pode vir a ressaltar sua relevância?

Em rápida análise, do caso brasileiro em particular, a ascensão em termos de popularidade dos jogos eletrônicos deu-se, particularmente, por volta da década de 1980. Se gerações anteriores estavam acostumadas às máquinas de pinball $\mathrm{e}$ fliperamas, toda uma nova leva de jogadores foi construída dentro do conforto do lar através da disseminação de consoles como o Atari e similares. Durante os anos 90, revistas especializadas em jogos eletrônicos e as populares locadoras de bairro ajudaram a alimentar um público ávido e apaixonado por esta plataforma de comunicação e, sobretudo, entretenimento. Console a console, uma geração após a outra (repleta de uma terminologia especializada em um sem-número de especificidades -- como plataformas de 8, 16, 32 bits; jogos para PC; mídias em cartucho, cd, dvd, blu-ray, etc), o jogador brasileiro contou ainda com uma facilidade em comparação ao primeiro mundo:

3 Trecho em tradução livre. No original: "Video games have been central to mass culture for more than twenty years, yet surprisingly few books today attempt a critical analysis of the medium", a de ser um mercado extremamente afeito aos produtos "piratas"; seja o cartucho de procedência duvidosa comprado em países vizinhos, seja aquele jogo conseguido via internet de maneira ilegal. Assim, a troca de informações e produtos tornouse pouco dispendiosa e consideravelmente mais facilitada.

Décadas depois, as crianças, e jovens que cresceram com isso, são hoje adultos o suficiente para contar, por intermédio de produção cultural e acadêmica, a história do impacto que esta linguagem teve em suas formações. Thomas Bissell (2010) e Michael W. Clune (2015), autores norte-americanos de literatura, puderam relatar maneiras como os videogames lhes influenciaram como ferramenta narrativa. Ambos escreveram, em anos recentes, um formato muito singular do gênero literário "memórias": envolveram suas próprias vivências pessoais -- o primeiro, dos primeiros anos de sua idade adulta; o segundo, percorrendo sua infância até o final da adolescência -- e os modos como suas narrativas pessoais estão fortemente atreladas a jogos de videogame. Esse impacto não se restringe em exemplos oriundos de primeiro mundo. Autores brasileiros, como Daniel Galera e Daniel Pellizzari já deixaram claro a influência da narrativa de jogos eletrônicos na literatura que praticam: o primeiro, com seu conto Virando o Jogo (2010) publicado originalmente no quarto número da Revista Serrote; o segundo, na caracterização de uma das personagens do romance Digam a satã que o recado foi entendido (2013).

Jogos eletrônicos podem, assim, ser analisados também dentro da própria esfera da literatura, mais especificamente na área de literatura comparada; uma que estimula o diálogo entre o ramo narrativo por excelência com outras áreas e contextos. Neste sentido, a quarta edição da revista Intersemioses (2013) do Núcleo de Estudos de Literatura e Intersemioses da Universidade Federal de Pernambuco (UFPE) foi inteiramente dedicada às 
interseções possíveis entre videogames e a literatura, numa edição em que foi reiteradamente salientada a importância de uma leitura multidipliscinar nas análises e na lida com estas novas mídias (TEIXEIRA, 2013, p. vi). Importante ressaltar, contudo, que basta uma rápida consulta ao sumário da publicação para notar a numerosa quantidade de artigos produzidos por autores estrangeiros, o que nos leva a crer se tratar de um campo que, no país, ainda não foi devidamente explorado dentro das possibilidades da academia. Assim, destaca-se não apenas a relevância desta colaboração à comunidade científica, mas sublinha-se também o fato de que há, infelizmente, pouca fortuna bibliográfica sobre o tema em português brasileiro.

As análises aqui dispostas, portanto, terão por base o ramo norte-americano do Game Studies e levará também em consideração fontes publicadas em revistas, jornais, documentários, etc. Para o presente trabalho, constitui intenção ulterior ressaltar como se dá a referenciação a elementos da cultura popular em um jogo do estilo adventure, bem como sublinhar formas como este hibridismo narrativo tem lugar em um jogo eletrônico. Esses artifícios de linguagem, tão próprias ao videogame, provam ser este um terreno adequado para uma análise que opte pelo usode umazonainterdisciplinar entre Literatura Comparada e Estudos da Mídia. Assim, atentaremo-nos neste trabalho por sobre o jogo Day of the Tentacle (DOTT), lançado originalmente em 1993 e parte do universo criado por sua produtora LucasArts. Procederemos, em um primeiro instante, à apresentação do gênero a que pertence este jogo, o adventure, apontando sua essência linear e de cunho narrativo. Em sequência, analisaremos a história de DOTT e a "linhagem" de jogos em que nosso objeto se insere, bem como certas particularidades de sua mecânica dentro de seu gênero. Por fim, ressaltaremos as intercessões narrativas que o jogo estabelece com a história norte-americana, personagens arquetípicos da literatura de horror e de ficção científica, desenhos animados e o cinema.

\section{O gênero adventure e seus caminhos narrativos}

Famoso durante quase toda a década de 1980, e atingindo o ápice de sua popularidade nos anos 90, os jogos estilo adventure foram bastante populares e ajudaram a sedimentar os computadores como um ambiente de divertimento eletrônico distinto daqueles que havia em fliperamas e consoles. Havia, nos computadores pessoais (PC, sigla para personal computer), um nicho para jogos que faziam uso do teclado e mouse; instrumentos que, à época, faziam parte apenas da mecânica de games que requeriam também raciocínio e certa estratégia, e não apenas reflexos rápidos e habildade no manuseio de um controle. Exemplos de títulos nesse estilo são Pirates! (1987) e Sim City (1989), que solidificaram a visão do computador como ambiente adequado para jogos que envolviam inteligência e pensamento estratégico.

O primeiro adventure de grande sucesso, King's Quest, foi lançado em 1983. A dinâmica de jogo deste título difere muito daquela que hoje se toma como intrínseca ao estilo: os gráficos apresentados no monitor ilustravam, sim, o desenrolar da aventura, porém o usuário deveria interagir com elementos que surgiam à tela através de entradas verbais inseridas via teclado, utilizando-se de uma lista pré-definida de ações possíveis presentes no manual impresso que acompanhava o produto.

A linguagem de interação dominante ao gênero é aquela do aponte-e-clique (point-andclick), na qual o jogador interage com os cenários à tela através do cursor do mouse. Esta dinâmica foi apresentada ao público, apenas em 1987, pela Lucas Arts, com Maniac Mansion -- título ao qual o jogo que nos deteremos neste trabalho, Day Of The Tentacle (1993) é sequência direta. 
O gênero adventure é, conforme se tenciona argumentar, focado na narrativa. Nela, o jogador avança através da solução de certos "problemas", quebra-cabeças (puzz̨les) surgidos no decorrer do jogo. Estes, raras exceções, poderão ser resolvidos através da interação com o cenário, outras personagens ou pelo manuseio de objetos angariados em um inventário adquirido durante a jornada. Para um jogo ser reconhecidamente detentor de qualidade entre os admiradores do gênero devem, segundo Karhulahti (2014, p. 213), obedecer a três características básicas dentro de sua ambientação: lógica, integração e expressividade.

Como em toda história, há a necessidade da criação de um universo ficcional particular. Mary Ann Buckles, em sua pioneira tese de doutoramento acerca das potencialidades da ficção em meios eletrônicos, ressalta a importância da narrativa ao gênero, ao afirmar que "se o jogador não entende a história, a história simplesmente estanca $^{4 \prime}$ (BUCKLES, 1985, p. 180). O que moveria o jogador adiante seria, em essencial, a vontade de progressão dentro do enredo, seu envolvimento com o material e a empatia pelas personagens, não um acúmulo de pontos e recordes (raríssimos adventures contabilizam pontos); não a competição com seu colega que está de posse do controle número dois (raríssimos adventures admitem mais de um jogador); não a disputa para tentar terminar mais rápido (ainda que se saiba a sequência correta e a solução de todos os puzzles, o gênero conta com títulos relativamente longos). Portanto, em questão de motivações para progredir ou experienciar jogos deste gênero, conta-se muito a paixão por uma história bem construída e ambientalizada. James Newman argumenta que jogos para PC, diferente daqueles para consoles, intentam, sobretudo, fazer "os usuários não apenas juntem-se aos atores no palco, mas tornem-se atores, abolindo a noção geral

4 Tradução livre. No original, "...if the player does not understand the story $[. .$.$] the story simply stops"$ de uma platéia"5 (NEWMAN, 2004, p. 101). E, a partir desta característica por demais proeminente aos jogos desse estilo, pode-se construir uma aproximação entre modalidades narrativas outras, como o cinema e os desenhos animados.

Day Of The Tentacle, objeto de análise do presente trabalho, foi o adventure que, em sua geração (pré-1995), mais evidentemente deixou-se influenciar por estas outras linguagens, influências que tentaremos ressaltar no decorrer do presente trabalho. A isto, colaborou o fato de este ser um dos primeiros jogos a se beneficiar de um maior arsenal multimídia: animações melhor apresentadas; música ambiente; efeitos sonoros que aprofundavam a sensação de imersão; foi, também, o primeiro jogo do estilo onde cada personagem tinha suas falas completamente dubladas, ao invés de simplesmente escritas na tela como em um roteiro. Todos esses avanços tecnológicos contribuiram para uma melhor efetivação das inferências que os criadores se propuseram a criar, uma vez que nos colocamos em consonância com Pignatari, enxergando este artifício semiótico como algo inerente à produção de desdobramentos de sentidos artísticos / literários / narrativos, ao afirmar que são “operações lógicas de digitalização para efeito de análise e controle de fenômenos contínuos, donde tendem a multiplicarse para maior precisão na apreensão das gradações desse mesmo contínuo" (2004, p. 61-2). A crítica especializada da época afirmou reiteradamente (HUTSKO, 1993; MAY, 1993) que o jogo mais parecia um desenho animado -- em virtude da plasticidade sonora, ambientalização, tom da narrativa, etc -- que um... jogo de videogame.

\section{O dia do tentáculo}

Em outubro do ano de 1987, investigações acerca do desaparecimento da jovem Sandy Pantz

5 Tradução livre. No original, "Computer users not only join the actors on stage, but become actors, abolishing the notion of audience altogether" 
conduziram à crença de que ela fora raptada pelo Dr. Fred Edison. A vítima estaria viva, mantida em cativeiro, e alojada em uma espécie de laboratório localizado nos porões da mansão onde Edison reside com sua mulher Edna e seu filho Ed. O estudante Dave Miller, namorado de Sandy, recruta então seis pessoas entre seu grupo de amigos, todos com características físicas distintas e personalidades muito particulares, na tentativa de formar uma força-tarefa para auxiliar no resgate de sua companheira. Em sua jornada, Dave e seu bando tiveram de, bravamente, enfrentar situações assustadoras e pouco convencionais: tentáculos roqueiros, corpos embalsamados, explosões de hamsters em microondas e outros cenários estapafúrdios num roteiro que mais se aproxima ao de um filme de comédia/terror de baixo orçamento do que um videogame.

Este, no entanto, é o enredo geral de Maniac Mansion, título que introduziu profundas inovações no cenário de jogos eletrônicos.

Aparte à imensa contribuição ao gênero pela introdução da dinâmica point-and-click já mencionada anteriormente, a criação da Lucas Arts, liderada pelos desenvolvedores e roteiristas Ron Gilbert e Gary Winnick, apresentou ainda outras inovações no gênero, tais como diversidade na escolha de personagens; possibilidade de desfechos diferentes a depender de com quais membros da turma o jogador alcança o fim do jogo; multiplicidade de caminhos "em aberto" para alcançar o cenário final (certas rotas, por exemplo, só poderiam ser alcançadas caso a "equipe" montada pelo jogador contar com um personagem de dada estatura ou tipo físico; de outra forma, um caminho diferente deverá ser tomado), etc. Desnecessário dizer que o jogo foi bastante bem recebido pelo público e crítica e, décadas depois, é ainda razão de interesse para a comunidade envolvida com jogos de adventure.

Contudo, apesar do grande sucesso e da adaptação a diversas outras plataformas de entretenimento eletrônico (inclusive, consoles caseiros, como o Nintendo Entertainment System), o jogo apenas obteve uma sequência cinco anos após seu lançamento. A data escolhida para o lançamento da esperada sequência de Maniac Mansion foi junho de 1993. E, desta feita, o vilão não seria mais a família Edison, que se resignara a apenas prosseguir, por um lado, com Dr. Fred e a pesquisa científica; por outro, com Edna e Ed, com outras atividades mundanas (a mansão tornara-se, agora, um pequeno e informal hotel, com aluguel de quartos para estadias curtas). $\mathrm{O}$ vilão passaria a ser uma certa personagem coadjuvante; residindo, aí, o motivo do título do jogo: agora, seria a vez dos tentáculos entrarem mesmo em cena.

Nos instantes iniciais do jogo podemos perceber que, mesmo trilhando um caminho o qual nos conduz ao retorno a um ambiente que nos é familiar -- a mansão dos Edison --, este é um cenário distinto àquele que jogadores até então estavam acostumados: há música, e tanto o som quanto as animações são fluidas e remetem ao estilo cartunesco. Aves voam no monitor do computador e uma adaptação, das suites Peer Gynt, do compositor norueguês Edvard Grieg, ressoa das caixas de som, os tentáculos têm vozes. Todas essas possibilidades são advindas do surgimento e da popularização de placas de som mais potentes e de diferentes mídias para o armazenamento e leitura de dados, como o CD-ROM. Day Of The Tentacle inaugura, assim, uma era de novas possibilidades tecnológicas para os jogos de adventure.

Em se tratando de dinâmicas narrativas estabelecidas, se cinco anos antes podíamos administrar o desenvolvimento do jogo através da escolha de personagens, aqui o jogador pode manusear, através de um fato inserido pelo próprio enredo geral, um outro elemento: o tempo.

Controlamos, aqui, três personagens: Laverne, Hoagie e Bernard. Bernard é o único dentre eles que estava presente na primeira visita à 
mansão em 1987. A princípio, controlamos apenas um de cada vez, ao gosto do próprio jogo. Depois, mais à frente, passamos a ser capazes de intercalar entre eles por escolha própria.

Certa noite, com Hoagie e Laverne em sua casa, Bernard recebe uma mensagem de seu amigo velho amigo Green Tentacle. Este avisa que está em apuros, pois Purple Tentacle havia sofrido uma mutação, tornando-se um gênio maligno e ambos foram trancados por Dr. Fred nos porões da mansão. Bernard, então, vai em busca de seu amigo. Após libertá-los (e, uma vez livre, Purple Tentacle sumir completamente), só então Bernard se dá conta de que Dr. Fred agora estava fazendo um bem: Purple havia perdido totalmente a razão e se transformado em um ser de maléficas propensões.

Dr. Fred sugere, então, que o trio participe de sua mais nova experiência: uma máquina do tempo chamada de Chron-o-John. A intenção é que as personagens retornem ao passado e impeçam a mutação de Purple Tentacle acontecer. Os três topam; contudo, um erro acontece e todos são dispostos no mesmo lugar (a mansão) em eras diferentes: Hoagie é jogado 200 anos no passado, Laverne 200 anos no futuro, enquanto Bernard permanece no presente. Felizmente, eles são capazes de trocar informações e enviar itens uns aos outros através do Chron-o-John. O jogador não tardará a perceber que certas ações efetuadas em cada uma das temporalidades acarretam algum tipo de mudança em outra, fazendo, então, que os puzzles atuem em três esquemas temporais -- passado, presente e futuro --, tornando-os muito mais complexos e interessantes. Day of The Tentacle também representa a sedimentação de uma linguagem muito particular dos adventures produzidos pela Lucas Arts: uma que fortalece um diálogo direto não apenas com outros jogos dentro de seu próprio universo mas com outras linguagens artísticas - como a música, cinema, desenhos animados e a própria literatura. Estes são alguns dos aspectos que tentaremos ressaltar a seguir.

\section{Possibilidades e intercessões}

A análise proposta visa refletir acerca de referências utilizadas na dinâmica do jogo, de modo que diretamente colaborem para sua efetividade narrativa.

Uma primeira relação que podemos estabelecer é a que diz respeito ao estilo visual do jogo, que mantem estreita relação estética aos desenhos do Looney Tunes, da Warner Bros. (no Brasil, o título ficou popular como "Pernalonga e Patolino"). Podemos perceber a semelhança já nos primeiros segundos de jogo: a música etérea, e os traços exagerados nas feições do pássaro quando da perda de fôlego, muito próximos daquilo que temos na referida série de TV.

O humor é marca do jogo, algo que percebemos em sua introdução: a temida mutação que Green Tentacle reporta a Bernard faz brotar não mais do que dois pequenos bracinhos em sua contraparte arroxeada. A expectativa na transformação não é efetivada e realiza-se, pois, na forma de humor.

É também marcante, em diferentes momentos do jogo, a história ser interrompida para dar lugar a rápidas animações em que o jogador pode tomar nota de que Purple Tentacle segue dando vazão às suas maldades mundo afora. $\mathrm{O}$ modo como o jogo procede com essas informações é, contudo, um tanto peculiar. Temos uma rápida pausa nas ações e, do fundo de uma tela preta, um jornal se aproxima e toma a tela. Passando-se rápidos segundos, o jornal "explode" ao monitor e podemos, enfim, ler as manchetes -- através dela, tomamos ciência do andamento de narrativas paralelas.

A utilização da própria ideia de mídia, para que ela mesma passe a contar as histórias, é muito 
utilizada em séries televisivas, cinema e histórias em quadrinhos. Citizen Kane (1941), de Orson Welles, foi um dos filmes que muito utilizou-se dessa técnica. Nas histórias em quadrinhos, a série The Dark Knight Returns (1986), de Frank Miller, utilizava reportagens de televisão para entendermos como se dava a disseminação da opinião pública às ações do homem-morcego. A mídia como próprio elemento narrativo, então, coloca outra esfera de apreciação da história -- a mesma que chega a nós está sendo narrada, também, a um publico distinto, fictício, por meio de outras fontes.

O jogo, especialmente nas falas da personagem Dr. Fred, também rechaça de diversas maneiras uma das características que Fredric Jameson (1991, p. 95), teórico norte-americano que se debruça acerca do tema da pós-modernidade, encara como uma das principais características de nosso tempo -- o rechaço à crença demasiada na infaliabilidade do discurso científico. Apesar de certas nuances em seu discurso, podemos perceber que ele, do alto de todo o seu conhecimento científico, não pode ser tido como um detentor de um discurso confiável e verdadeiro. O jogo explora isso de uma maneira abertamente cômica. Podemos ver, a título de exemplo, nas imagens a seguir o modo como ele "explica" o fato de sua máquina do tempo conseguir enviar objetos de uma temporalidade à outra.

Aliás, a própria máquina do tempo de Dr. Fred já é algo risível: podemos perceber, pelas fotos, que elas são cabines de mictório. Daí, talvez, derive o seu nome "Chron-o-John", trocadilho intraduzível que envolve Chronos (o deus grego do tempo), "Chromossome" (cromossomo, em língua inglesa) e a inclusão de "John", que é uma forma muito impessoal e popularesca de se referir a banheiro em língua inglesa.

Uma das grandes inovações apresentadas pelo jogo foi a da interação entre três espectros temporais: passado, presente e futuro, anteriormente explicitados. As ações efetuadas, em cada uma dessas três esferas, podem acarretar mudanças em uma outra, o que torna as dinâmicas dos puzzles bem mais rebuscada. No entanto, fora da esfera dos jogos de adventure, esta não é uma dinâmica tão nova assim: já foi bastante explorada, por exemplo, no cinema. O popular filme Back to the Future (1985) é um exemplo. Quem não se lembra do primeiro episódio da série de três filmes, onde Marty McFly quase acaba fazendo com que sua mãe se apaixonasse por ele mesmo? Dr. Brown avisa-lhe de que isso não é possível que aconteça, pois, do contrário, ele nunca nasceria e as esferas temporais entrariam em colapso.

Quanto a estas tipologias, presentes em outras mídias, convém lembrarmos dos estereótipos de cada uma das personagens: o nerd (Bernard), uma estudante "maluquinha" (Laverne) e um roqueiro (Hoagie), cada um deles bastante famosos no cinema e em séries de televisão. Podemos, também, listar a figura do cientista maluco em Dr. Fred e da bruxa malvada em sua esposa, Edna.

Várias são, portanto, as referências possíveis que circundam e dinamizam o jogo. Estas atuam como elementos narrativos de forma a estruturar melhor o enredo.

\section{Algumas considerações}

Jogos eletrônicos, em especial aqueles focados em narrativas, podem contribuir para que novas modalidades de texto possam ser produzidas, desempenhadas e consumidas. A capacidade que os videogames têm, muito em virtude de seu potencial multimídia, de imiscuir e hibridizar formas diversas de discursos e linguagens (o cinematográfico, quadrinístico, literário, histórico, etc., conforme ressaltamos no presente trabalho) entra, pois, em consonância com aquilo que o teórico cultural Douglas Kellner ressalta como característico do discurso de um "crepúsculo Moderno", em seu 
ardor pelo rompimento com o passado e um pendão à combinação para as novas produções culturais (KELLNER, 1995, p. 232). O resultado híbrido e peculiar de Day of the tentacle atesta a potencialidade e a pertinência desta plataforma no esquema da produção de narrativas.

Narrativas estas que, tal como outras desenvolvidas em áreas a que estamos devidamente habituados na academia (o Literário, o Fílmico), estão em constante avanço, de forma a gerar suas próprias particularidades. Nos videogames, a aproximação entre modalidades narrativas diversas está cada vez mais relativa. A respeito de jogos mais modernos que mantêm estreita relação com enredos (como é o caso de Fallout 3 e Fallout New Vegas), Scheppler (2012) fala em uma diegese própria, na medida em que games mais recentes são já capazes de apresentar um universo diegético dotado de tantos elementos a contribuir para uma imersão (cidades inteiras para interagir, dotadas de casas, personagens, etc) que a participação do jogador é solicitada de modo que o fio narrativo central pode vir a se tornar secundário -- e, a depender do jogo, nem é necessário ser acompanhado e/ ou completado. Outra característica particular nos é sublinhada por Lindley (2002), ao comparar a diferença entre o conflito na narrativa clássica e no videogame: naqueles, o conflito é estabelecido no começo da narrativa, suas implicações aparecem ao longo dela e ao final temos sua resolução; no videogame, Lindley argumenta que o conflito tende a se estabelecer e ser resolvido pelo jogador no 2 " “ato", ao passo em que os demais geralmente "escapam" ao controle do jogador.

No que diz respeito ao jogo aqui analisado, sua relevância ainda permanece. Fora de catálogo desde os anos de 1990, jogá-lo em sistemas operacionais atuais é problemático, no entanto, uma edição remasterizada com gráficos atualizados e comentada pelos roteiristas e produtores foi lançada em março de 2016. Day of the tentacle foi, também, colocado no topo da lista de melhores jogos do gênero pelo site especializado Rock, Paper, Shotgun em matéria que data de junho de 2015. Nela, o crítico John Walker afirma que se trata de um jogo "afiado, espirituoso, inteligente e enormemente satisfatório. DOT'T permanece como o livro-base pelo qual designers de jogos de adventure deveriam estudar antes de pensar em conceber um quebracabeça". ${ }^{6}$

\section{Referências}

BACK TO THE FUTURE. Dir. Robert Zemeckis. Universal Pictures, 1985 [produção]. 1 DVD (116 $\min )$

BISSELL, T. C. Extra lives: why video games matter. New York: Pantheon Books, 2010.

BUCKLES, M.A. Interactive fiction: the computer storygame 'Adventure'. 1985. Tese (Doutorado em Literatura). University of California.

CITIZEN KANE. Dir. Orson Welles. RKO Pictures, 1941 [produção]. 1 DVD (119 min)

CLUNE, M. W. Gamelife: a memoir. New York: Farrar, Straus and Giroux, 2015.

DAY OF THE TENTACLE. Prod. por Dave Grossman e Tim Schaffer. LucasArts, 1993.

GALERA, D. Virando o jogo. Porto Alegre: LDM 2.0, 2010.

GALLOWAY, A. R. Gaming: essays on algorithmic culture. Minneapolis: University of Minnesota Press, 2006.

HUTSKO, J. Talking tentacle's tyranny IN PC World. October, 1993

JAMESON, F. Postmodernism, or the cultural logic of late capitalism. Durnham: Duke University Press, 1991.

6 Tradução livre. No original, "it's sharp, witty, clever, and enormously satisfying. DOTT remains the textbook which all adventure game designers should study before they even consider conceiving a puzzle". 
KARHULAHTI, V. Fiction puzzle: storiable challenge in pragmatist videogame aesthetics IN Pbilosophy \& Technology. Number 27. pp 201-220. 2014.

KELLNER, D. Media culture: cultural studies, identity and politics between the modern and the postmodern. New York: Routledge,1995

KING'S QUEST. Prod. por Roberta Williams. Sierra, 1983.

LINDLEY, C.. The gameplay gestalt, narrative, and interactive storytelling. In: Proceedings of Computer Games and Digital Cultures Conference. Frans Mäyrä.

Tampere: Tampere University Press, 2002. Pp. 203-15. Disponível em www.digra.org/dl/ db/05164.54179.pdf.

MAY, S. A. Day of the tentacle - review IN Compute! Vol. 15 Issue 12, p118. December 1993.

MILLER, F. The Dark Knight Returns. New York: DC Comics, 1997.

NEWMAN, J. Playing with videogames. New York: Routledge, 2008.

NEWMAN, J. Videogames. New York: Routledge, 2004.
PELLIZZARI, D. Digam a satã que o recado foi entendido. São Paulo: Companhia das Letras, 2013.

PIRATES! Prod. por Sid Meyer. MicroPose, 1987.

SCHEPPLER, G.. Les formes d'adresse au spectateur: du cinéma au jeu vidéo de rôle, en passant par la bande dessinée. In: MEI (Médiation et Information). N. 34, Paris: L'Harmattan, 2012, pp. 145-56. Disponível em http://www.mei-info.com/ wp-content/uploads/2015/03/MEI_34_11.pdf. Acesso em 07 de Março de 2017.

SIMCITY. Prod. por Will Wright. Electronic Arts, 1989.

SMITH, R. Rogue leaders: the story of Lucas Arts. San Francisco: Chronicle Books, 2008.

TEIXEIRA, L. F. B. Figur@ções: games e literatura electrônica IN Intersemiose. Recife, Ano 2, N. 04. JulDez 2013.

WALKER, J.The 25 best adventure games ever made IN Rock, Paper, Shotgun. Disponível em: https:// www.rockpapershotgun.com/2015/06/12/bestadventure-games/7. Acesso em 08 de março de 2016.

Submissão em: junho de 2017

Aceito em: março de 2018 